\title{
Tracking Activities of Daily Living in the Home Office
}

\author{
Ju WANG ${ }^{1}$, Marcel AGUIRRE MEHLHORN, Katharina DITLER, \\ Benjamin EFFNER and Thomas M. DESERNO \\ Peter L. Reichertz Institute for Medical Informatics of TU Braunschweig \\ and Hannover Medical School
}

\begin{abstract}
Due to the COVID-19 pandemic, home-office has turned to be a common practice in many companies to limit physical contact to reduce the rate of infections in the workplace. To quantify office workers' ADLs, this work demonstrates unobtrusive monitoring of activities of daily living (ADLs) of an office worker in a home-office environment with three low-cost sensors: an accelerometer and two light sensors. We extract four elementary events: distinct and fain chair movement, monitor, and fridge usage, from which we derived seven ADLs using predefined rules. This simple system can support the quantification of ADLs of home-office workers.
\end{abstract}

Keywords. Activities of Daily Living, home-office, COVID-19 pandemic, sensor, AAL

\section{Introduction}

The COVID-19 pandemic has changed our lives in many ways. With the availability of telecommunication platforms, it is feasible to conduct much work without being at the office. To limit physical contacts and reduce infection rate in the workplace, home-office has turned to be a common practice in many companies. However, home-office could negatively influence office workers. For instance, the work-life balance can be disturbed to change the office workers' physical and mental well-being [1].

The term activities of daily living (ADLs) is defined as the self-care activities that are important for health maintenance and independent living [2]. ADL is an indicator of health status as performing ADLs depends on cognitive (e.g., reasoning, planning), motor (e.g., balance, dexterity), and perceptual (including sensory) abilities [3]. With the advances in sensor technology, big data, and artificial intelligence, low-cost sensors can implement, when installed at proper locations, physiological monitoring and functional monitoring [4] for certain subjects, e.g., the elderly or patients with certain disabilities. For instance, with motion sensors and contact sensors, Kaye et al. recorded Alzheimer patients' daily life [5]. Similarly, our previous work profiled the hip fracture patients' behavior after discharge with multimodal Internet of things (IoT) sensors such as accelerometer, motion, vibration, and contact sensors [6][7]. The Massey University Student Enterprise (MUSE) group tracked the toilet use of an elderly person with a light

\footnotetext{
${ }^{1}$ Corresponding author, Peter L. Reichertz Institute for Medical Informatics, Muehlenpfordstr. 23, 38106 Braunschweig, Germany; E-mail: ju.wang@plri.de.
} 
sensor in the rest room [8]. However, home-office has not yet been monitored by such simple systems.

Inspired by the related work of monitoring the ADLs of the elderly and the patients, this work will demonstrate unobtrusive ADL monitoring of an office worker with low cost and simple out-of-the-shelf sensors. We also showcase the information that can be extracted from the sensor data.

\section{Methods}

\subsection{Sensor setup}

We used a sensor kit (PLUX Biosignalsplux, PLUX wireless biosignals, S.A., Lisboa, Portugal) with sensors and hubs for data collection [9]. We used only three sensors (Fig. 1):

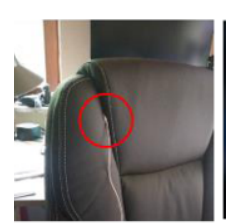

(a) ACC

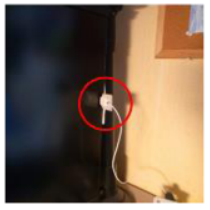

(b) LUX1

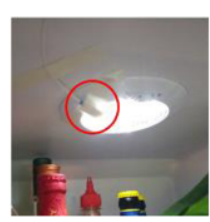

(c) LUX2

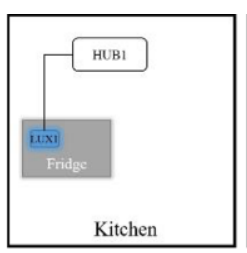

(d) Overview of sensor deployment

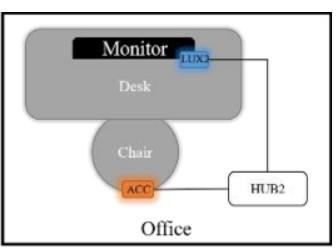

Figure 1. Hardware setup.

- An accelerometer (ACC) attached to the headrest of the office chair tracks the movement of the office worker (Fig. 1-a).

- A light sensor (LUX1) orthogonally attached to the monitor of the computer measures the display activity (Fig. 1-b).

- A light sensor LUX2 attached next to the refrigerator light checks if the refrigerator is open (Fig. 1-c).

We cabled ACC and LUX1 to a hub (HUB1) that then with Bluetooth connected to a smartphone (SF1), and LUX2 via another hub (HUB2) to another smartphone (SF2), which was placed near the fridge, as the computer was not within the Bluetooth reach from HUB2.

Our sensors recorded data over four weekdays. The system was started each day at about 07:00 and shut down at about 17:00. We configured the sampling rate for all sensors to $1 \mathrm{~Hz}$.

\subsection{ADL event extraction}

We divided the ADL event extraction from raw sensor data into two stages: elementary event and ADL events. We defined and extracted four types of elementary events:

1. Distinct chair movements are caused by evident activities like standing up or sitting down in the office chair. We employed Butterworth and median filters to smooth the data and an empirical threshold to detect distinct movement.

2. Faint chair movements are the slight movements at the workplace while the person remains in the chair. 
3. Monitor on/off event is derived by the light sensor, which illustrates clearly ascending and descending slopes when the monitor turns on and off, respectively. We applied an offset to the sensor data due to cope with interferences from the surroundings and ambient light.

4. Fridge open/closed event is detected directly from the signal as there is nearly no interfering light when the fridge is closed.

Based on the four types of elementary events, we extracted seven ADL events based on the social rhythm metrics (SRM) [10]. Table 1 lists the rules.

Table 1. Event extraction rules

\begin{tabular}{|c|c|c|c|}
\hline ID & ADL & Rule & Timeslot \\
\hline 1 & Begin of the working day & Distinct chair movement + monitor on & 07:00-08:00 \\
\hline 2 & Breakfast & $\begin{array}{l}\geq 2 \times \text { distinct movement chair }+ \text { monitor off }+ \text { fridge } \\
\text { open/closed }\end{array}$ & $08: 00-11: 00$ \\
\hline 3 & Concentration phase & Faint movement chair + monitor on & $07: 00-17: 00$ \\
\hline 4 & Lunch & $\begin{array}{l}\geq 2 \times \text { distinct chair movement }+ \text { monitor off }+ \text { fridge } \\
\text { open/closed }\end{array}$ & $11: 00-14: 00$ \\
\hline 5 & Afternoon Snack & $\geq 2 \times$ distinct chair movement + fridge open/closed & $14: 00-17: 00$ \\
\hline 6 & General working break & $\geq 2 \times$ distinct chair movement + monitor off & $07: 00-17: 00$ \\
\hline 7 & End of the working day & Distinct chair movement + monitor off & $16: 00-17: 00$ \\
\hline
\end{tabular}

\section{Results}

We obtained the elementary events. A sample set of events of a certain day is shown in (Fig. 2-(a)) with their starting- and ending-timestamps. Furthermore, after the event extraction, we can derive the target ADL events (Fig. 2-(b)) applying our rule set (Table 1). To quantify the office worker's ADL, we provide a statistical summary (Table 2).

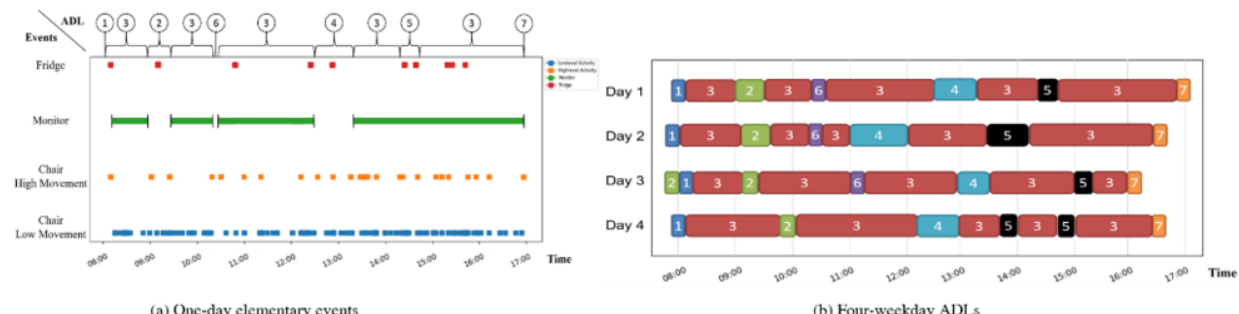

Figure 2. Elementary and ADL events. The IDs of the ADL events are at the top of the graph.

Table 2. Summary of ADL events in four weekdays

\begin{tabular}{llll}
\hline ID & ADL & Average time & Average timeslot \\
\hline 1 & Begin of the working day & - & $07: 45-08: 15$ \\
2 & Breakfast & ca. $20 \mathrm{~min}$ & $09: 00-10: 00$ \\
3 & Concentration phase & ca. $90 \mathrm{~min}$ & $08: 00-09: 00$ \\
& & & $09: 30-11: 00$ \\
& & & $14: 00-16: 30$ \\
4 & Lunch & ca. $45 \mathrm{~min}$ & $11: 00-13: 30$ \\
5 & Afternoon Snack & ca. $15 \mathrm{~min}$ & $13: 30-15: 15$ \\
6 & General working break & ca. $10 \mathrm{~min}$ & $10: 30-11: 15$ \\
7 & End of the working day & - & $16: 00-17: 00$ \\
\hline
\end{tabular}




\section{Discussion}

Our experiment demonstrated that applying a limited number of low-cost and out-of-theshelf sensors is feasible to track ADLs of workers in home-office. We designed the sensor deployment carefully to monitor the ADLs in an unobtrusive manner. Our data processing pipeline is easy to implement.

Our results suggest that the monitored office worker's lifestyle was relatively stable within the monitored duration. There was barely any movements during the workday besides standing up to get some food. The obtained information may offer tips for healthy lifestyle. For example, to keep in work-life balance, people need to break more frequently during the workday [11].

In this work, we defined the ADL events according to the SRM. On the one hand, we showed the potential of automatically collecting data for the SRM using sensor technology. On the other hand, the sensor data can be used to verify the precision of the SRM diary, which is usually collected by manually survey with the subjects.

The variety and the amount of sensors in the demonstrated system limit the ADL types that can be detected. The system cannot distinguish the work-relevant and nonrelevant activities when the worker is in the chair. The system cannot detect the activies out of the sensing scope either, e.g., the phone calls with colleagues in a different room.

In future work, additional data from more test persons with more sensors can be derived to broaden our results over an extended period. In particular, we intend to include phone calls performed with wireless devices. Furthermore, a smartwatch with an accelerometer or motion sensor can be used to track a person's movements at home to verify the findings of the accelerometer on the office chair.

\section{References}

[1] Majumdar P, Biswas A, Sahu S. COVID-19 pandemic and lockdown: cause of sleep disruption, depression, somatic pain, and increased screen exposure of office workers and students of India. Chronobiol Int. 2020;37(8):1191-200.

[2] Troyer AK. Activities of daily living (ADL). Encycl Clin Neuropsychol. 2011;28-30.

[3] Mlinac ME, Feng MC. Assessment of activities of daily living, self-care, and independence. Arch Clin Neuropsychol. 2016;31(6):506-16.

[4] Wang J, Spicher N, Warnecke JM, Haghi M, Schwartze J, Deserno TM. Unobtrusive health monitoring in private spaces: the smart home. Sensors. 2021 Jan 28;21(3):864.

[5] Kaye JA, Maxwell SA, Mattek N, Hayes TL, Dodge H, Pavel M, et al. Intelligent systems for assessing aging changes: home-based, unobtrusive, and continuous assessment of aging. J Gerontol B Psychol Sci Soc Sci. 2011;66B:i180--i190.

[6] Marschollek M, Becker M, Bauer JM, Bente P, Dasenbrock L, Elbers K, et al. Multimodal activity monitoring for home rehabilitation of geriatric fracture patients-feasibility and acceptance of sensor systems in the GAL-NATARS study. Informatics Heal Soc Care. 2014;39(3-4):262-71.

[7] Wang J, Bauer J, Becker M, Bente P, Dasenbrock L, Elbers K, et al. A novel approach for discovering human behavior patterns using unsupervised methods. Z Gerontol Geriatr. 2014;47(8):648-60.

[8] Moretti G, Marsland S, Basu D, Sen Gupta G, The MUSE Group. Towards a monitoring smart home for the elderly: one experience in retrofitting a sensor network into an existing home. J Ambient Intell Smart Environ. 2013 Jan 1;5(6):639-56.

[9] biosignalsplux - PLUX Store [Internet]. [cited 2021 May 10]. Available from: https://plux.info/12biosignalsplux

[10] Monk T, Flaherty J, Frank E, Hoskinson K, Kupfer D. The Social Rhythm Metric: an instrument to quantify the daily rhythms of life. J Nerv Ment Dis. 1990;178(2).

[11] Roche - 20 tips for maintaining a healthy work-life balance [Internet]. [cited 2021 Sep 8]. Available from: https://www.roche.com/careers/our-locations/asia/india/service/folder/20_tips_for_maintain.htm 